\title{
El Día de la Luz (the Day of Light): two hours optics demonstration for secondary school students
}

Osamu Takayama, Armand Niederberger, Petru Ghenuche, Manoj Mathew, Giovanni Volpe

Osamu Takayama, Armand Niederberger, Petru Ghenuche, Manoj Mathew, Giovanni Volpe, "El Día de la Luz (the Day of Light): two hours optics demonstration for secondary school students," Proc. SPIE 9665, Tenth International Topical Meeting on Education and Training in Optics and Photonics, 96650K (3 June 2007); doi: 10.1117/12.2207500

SPIE Event: Tenth International Topical Meeting on Education and Training in Optics and Photonics, 2007, Ottawa, Ontario, Canada 


\title{
El día de la luz (the day of light)-two hours optics demonstration for secondary school students
}

\author{
Osamu Takayama, Armand Niederberger, Petru Ghenuche, \\ Manoj Mathew and Giovanni Volpe \\ ICFO-The Institute of Photonic Sciences, Mediterranean Tecbnology Park, \\ Av. del Canal Olimpic s/ $n 08860$ Castelldefels (Barcelona), Spain \\ +34 935534046, +34 935534000(FAX), osamu.takayama@icfo.es
}

\begin{abstract}
In many countries the potential of optics as an exciting part of science is not fully exploited in highschool education. In addition, optics is often not taught in relation with daily experiences. With the motivation to expose the potential of doing otherwise to motivate students, we developed and implemented a two hour-long hands-on introduction to optics for high school students. We termed the program: The Day of Light. By attending the program, students learn basic concepts such as polarization, wavelength, color, stereoscopic vision, reflection and refraction in connection to everyday experiences based on applications of optics. The demonstration was fully organized and carried out by the ICFO Ph.D students who were members of the ICFO Optical Society of America (OSA) Student Chapter.
\end{abstract}

\section{Introduction}

Optics is often not taught in relation with daily experiences at high school level although optics is very visual and therefore can attract students. Moreover, many students do not appreciate the importance of optics even though they use it extensively in daily life. This is probably due to the intense curriculum and little time to prepare some attractive demonstration of optics. Moreover, high school physics instructors may have little background in optics because their background varies from physics to biology and they may have to teach a science field other than their background in high school.

With motivation to expose secondary school students to the fascinating field of optics using daily applications, we developed and implemented a two hour-long hands-on introduction to optics for high school students. Previously, there have been introductory workshops for elementary school students by other groups [1]. We named the program: El D ía de La Luz (The Day of Light) [2]. In this workshop, we cover basic concepts of ight, namely, polarization, refraction, reflection, and stereoscopic vision. In the selection of topics for demonstration, we particularly pay attention to applications and examples that students use and see everyday, such as liquid crystals used for the display of their mobile, CDs, perception of mages in three-dimensions. We either visited the local high school or invited students and their teacher to our institute for our program. Those workshops were organized and conduced by the Ph.D students in the ICFO Optical Society of America (OSA) Student Chapter.

\section{ICFO-OSA student chapter}

Our OSA student chapter at ICFO based on Barcelona, Spain was founded in 2004 [3]. ICFO-OSA student chapter currently embraces 30 post-graduate students from over 15 countries. Since its initiation, we organized bi-weekly seminar by students in ICFO which became weekly colloquium from 2007, and invited some distinguished speakers in the fields of optics, such as Dr. Emil Wolf, Dr. Joseph Eberly, and Dr. Chris Dainty as our internal activities within our institute. Very recently, we organized and hosted the first exchange program among OSA student chapter in Europe where three other chapters from Southampton (UK), Munich (Germany), and Naples (Italy) participated. This initiative is to exchange research interests, get acquainted with other research institute, and establish a network. One of the most important out-reach activities in our OSA student chapter is to introduce optics to secondary school students. Through these activities, we earned the prize, the Excellence Awafrbm the OSA in year 2006.

\section{El día de La Luz (The Day of Light) - Two hours optics demonstration}

In this section, we present the typical format of our two-hour workshops on light. Usually, 40 students aged from 15 to 18 together with several high school instructors are included in one session. Prior to the presentation, we give

Tenth International Topical Meeting on Education and Training in Optics and Photonics, edited by Marc Nantel, Proc. of SPIE Vol. 9665, 96650K · @ 2007 SPIE, OSA, IEEE, ICO doi: $10.1117 / 12.2207500$ 
students leaflets that summarize our demonstrations as shown in Figure 1 so that they could preview the main points of the workshop and high school teachers could learn the demonstration as well. As shown in Figure 2, we start with 10 minutes talk by using slides and posing students some fundamental questions such as 'where does light come from?' and 'what do we use it for?' in order to let students think about light sources such as the sun, light bulb, fire, etc. Then, we introduce basic concepts of light such as:

(1) Light can be described as a form of wave.

(2) Thus, it has the length of one cycle of this wave, called 'wavelength.'

(3) This wavelength corresponds to a color in the visible.

(4) Also as light is a form of wave, it has certain plane where the wave oscillates, called 'polarization.'

Then, we ask the students three everyday life questions about light to prompt students to think about optics before the hands-on experiments.

(1) How does the display of your mobile phone work?

(2) How do we guide light?

(3) Why is it handy to have two eyes?

So as to answer these questions, students go through three sessions of our experiments where each session is 20 minutes of hands-on experiments. Each session is conducted by two volunteers, and we switch the groups.

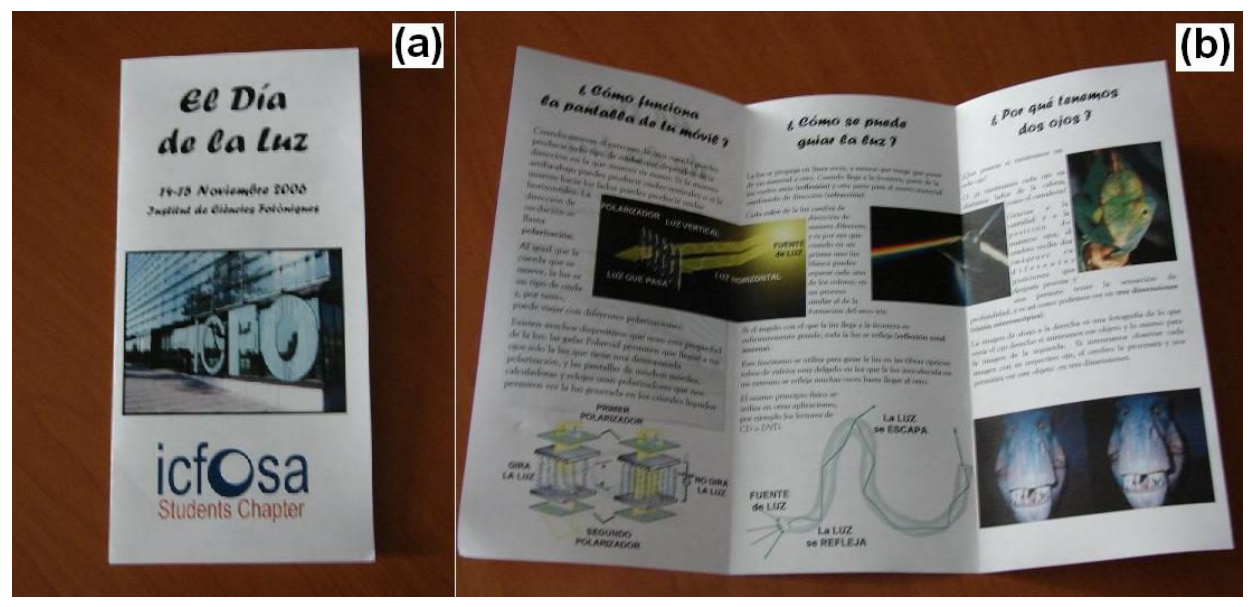

Figure 1: (a) and (b) A brochure of our program given to students.

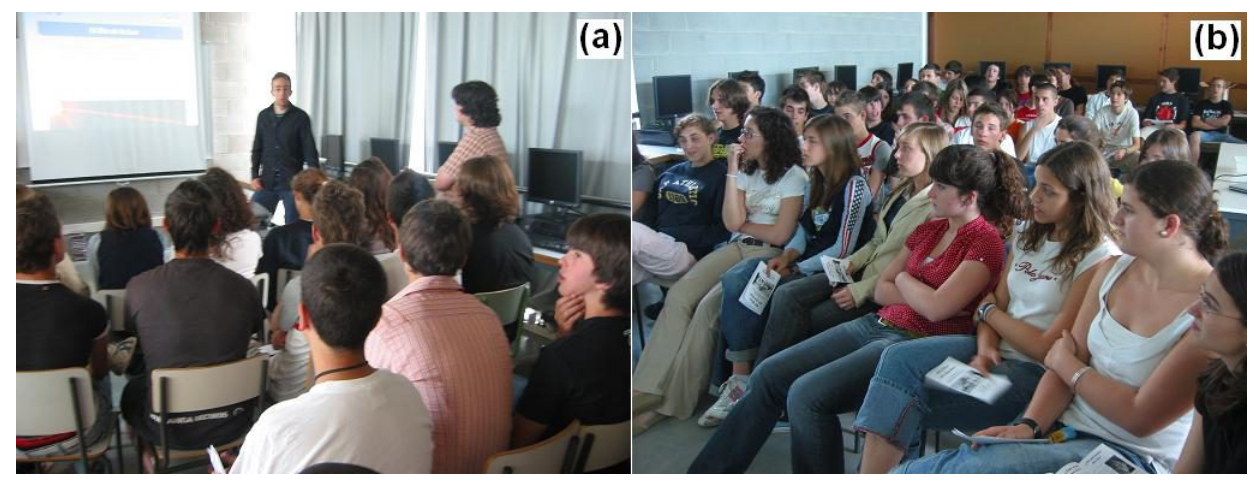

Figure 2: (a) and (b) Presentation prior to experiments. 
Table 1: Materials for the experiment of polarization and liquid crystal.

\begin{tabular}{|l|c|}
\hline Materials & number \\
\hline Plastic polarizer & 2 \\
A box with white light source and a polarizer & 1 \\
Liquid crystal cell & 1 \\
liquid crystal kit from OSA Rochester section & $50[5]$ \\
\hline
\end{tabular}

\subsection{Polarization and liquid crystal}

First of all, the polarization of light is explained using a few slides. Then, we also show a plastic polarizer to students and explain why the polarizer looks dark. In general, light from the sun or light bulb is not polarized and has a variety of polarizations. The plastic polarizer lets one polarization of light pass, and reflects light with other polarizations. With two polarizer perpendicular to each other, we demonstrate that light is completely reflected, and therefore it looks dark. We pass the polarizers around so that students can play with them as shown in Figure 3.
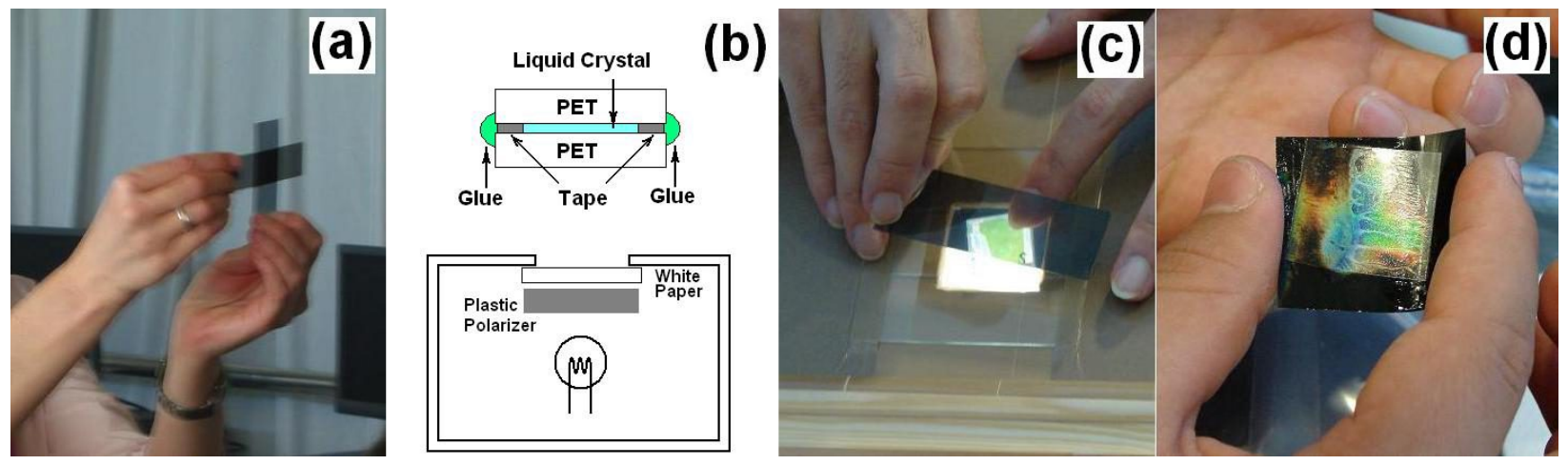

Figure 3: (a) Two plastic polarizers. (b) Structure of LC cell (above) and a box that emits polarized white light (below). (c) Demonstration of liquid crystal cell with a polarizer on top. (d) Students make their own liquid crystal cell.

Before discussing liquid crystal (LC) displays, we emphasize that liquid crystals are everywhere and that in fact we use them in the display of mobiles, laptops, calculators, watches and so on. With a drawing in a slide, we show that liquid crystals consist of long molecules in liquid, and with application of voltage or heat one can align them in such a way that they transmit or reflect light with certain polarization and wavelength of light.

While showing a slide that presents the structure of a LC, we show a LC cell. As shown in Figure 3 (b), this LC cell has E7LC [4] sandwiched by $2 \times 2 \mathrm{~cm}$ polyethylene terephthalate (PET) plates, and is glued to keep the LC between the plastic plates. When the cell is placed on the light source with one polarization, still the cell looks transparent. However, upon placing a polarizer on the other side of the LC cell, it shows different colors depending on the angle of the polarizer in the plane parallel to that of LC cell as shown in Figure 3 (c). Students learn that due to the orientation of LC molecules the polarization is rotated differently for different colors or wavelength. Thus one can change colors by changing the angle of the polarizer. Moreover, we explain that in LC displays, each pixel, which corresponds to a LC cell, changes its color by voltage instead of rotating the polarizer. This is because voltage changes the orientation of the LC molecules, which rotates the polarization of light with specific color, and enables us to extract specific colors by tuning voltage.

After a talk, we give students LC kits provided by the OSA Rochester section [5]. Students make their own LC cells that change their color depending on the temperature to understand that a certain temperature can orient the LC to reflect certain colors of light. They are amused and excited by learning what a LC is and how it works. They put the cell on their hands and compare the difference of temperature on their body with that of other students. The tools used for this demonstration are listed in Table 1. 


\subsection{Reflection and refraction}

With a triangle prism, we demonstrate refraction and reflection of light (Figure 4 (a)). When red light from a laser pointer is directed to the prism, students witness the light is bent the interface of air and prism, and thus they learn about the concept of refraction and the refractive index of materials. Moreover, depending on the angle of incidence, light that travels in the prism passes through the other side of the prism and goes out, or the light is reflected at the other interface of the prism. In this manner, the total-internal reflection of light is demonstrated in a simple experiment.

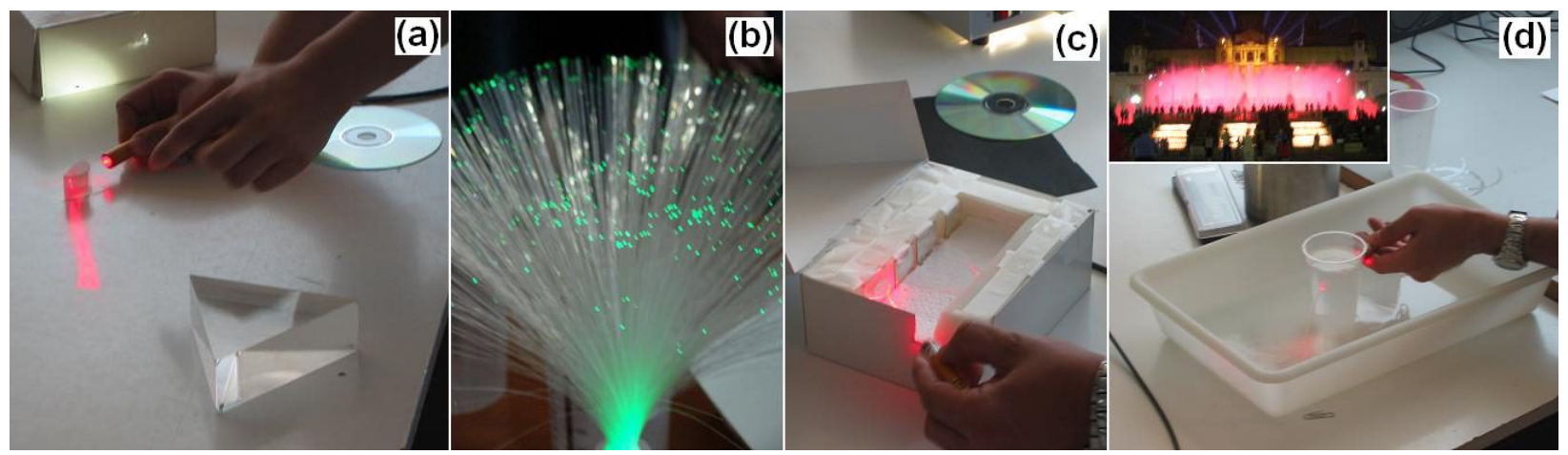

Figure 4: (Color images) Reflection and refraction of light by (a) a laser pointer and prisms, (b) optical fibers, (c) a box with mirrors, and (d) a cup with water and the inset is the fountain of Montjuic.

After understanding reflection and refraction, students are exposed to various applications of the phenomena. As in Figure 4 (b), we present optical fibers that connect long-haul communication system in which the data that encodes their voice may be carried as a form of light signal when they call someone in the North America from Europe. We stress that information in internet is carried in the same way as well. In order to understand the guiding of light by total-internal reflection, we let student play with a laser pointer and a foam box that has a hollow space with mirrors on both sides of the hollow space as shown in Figure 4 (c). With this simple gadget, the guiding of light by a series of reflection is visualized for students, and they learn how light from one end of the fibers propagates and comes out from the other end of the fibers.

We also perform an experiment on the guiding of light in flowing water. A plastic cup with a small hole in which water comes out is used. One of our volunteers shines a laser pointer toward the hole and demonstrates the red light of the pointer follows the path of flowing water. They are also explained that the fountain of Montjuic (See the inset in Figure 4 (d)), a famous touristic spot in Barcelona where the water of the fountain changes its color with music, works in the same principle as fiber optic cables.

As another everyday application of reflection, a volunteer explains optical data storage, such as CDs and DVDs that utilize reflection of laser to read information from the disc. Students learn that there are pits on disc surface which reflect laser to the detector in a different way depending on the shape and location of pits. In this manner information from the disc is optically read in terms of the sequence of the pits. The required kit for this session is shown in Table 2.

\subsection{Stereoscopic vision}

We perceive 3D image as superposition of two slightly different images obtained from both eyes. With one eye it is hard to obtain information about the distance of the image, though we can see the image in 2D. To demonstrate this concept, one volunteer asks a student to close one of his or her eyes and try to touch the finger of the volunteer from the side. Most of the students find themselves unable to touch the finger of the volunteer, and realize that it is hard to perceive the distance to the finger with one eye. Moreover, a volunteer asks a student to look at two rings of metal wire with one eye as in Figure 5 (a) and to tell which ring, either small or bigger one, is closer to the student, which turns out to be quite challenging to distinguish with one eye. Thorough those experiments, students realize that it is handy to have two eyes in order to comprehend the sense of distance.

Then, each student receives 3D images and 3D glasses. In the easiest form, these 3D pictures are essentially two blue and red pictures that are put together but slightly shifted when they are printed on a sheet of paper. Suppose the 
Table 2: Materials for the experiment of polarization and liquid crystal. Note that optical fiber cables do not necessarily have to be the ones for telecommunication. For demonstration purpose, optical fibers for decoration are sufficient.

\begin{tabular}{|l|c|}
\hline Materials & number \\
\hline Triangular or semi-cylindrical prism & 1 \\
CD or DVD & 1 \\
A box with mirrors & 1 \\
Optical fiber cable & $20-30$ \\
Plastic cups with a hole and a bucket with water & $2-3$ \\
Red laser pointer & 1 \\
\hline
\end{tabular}

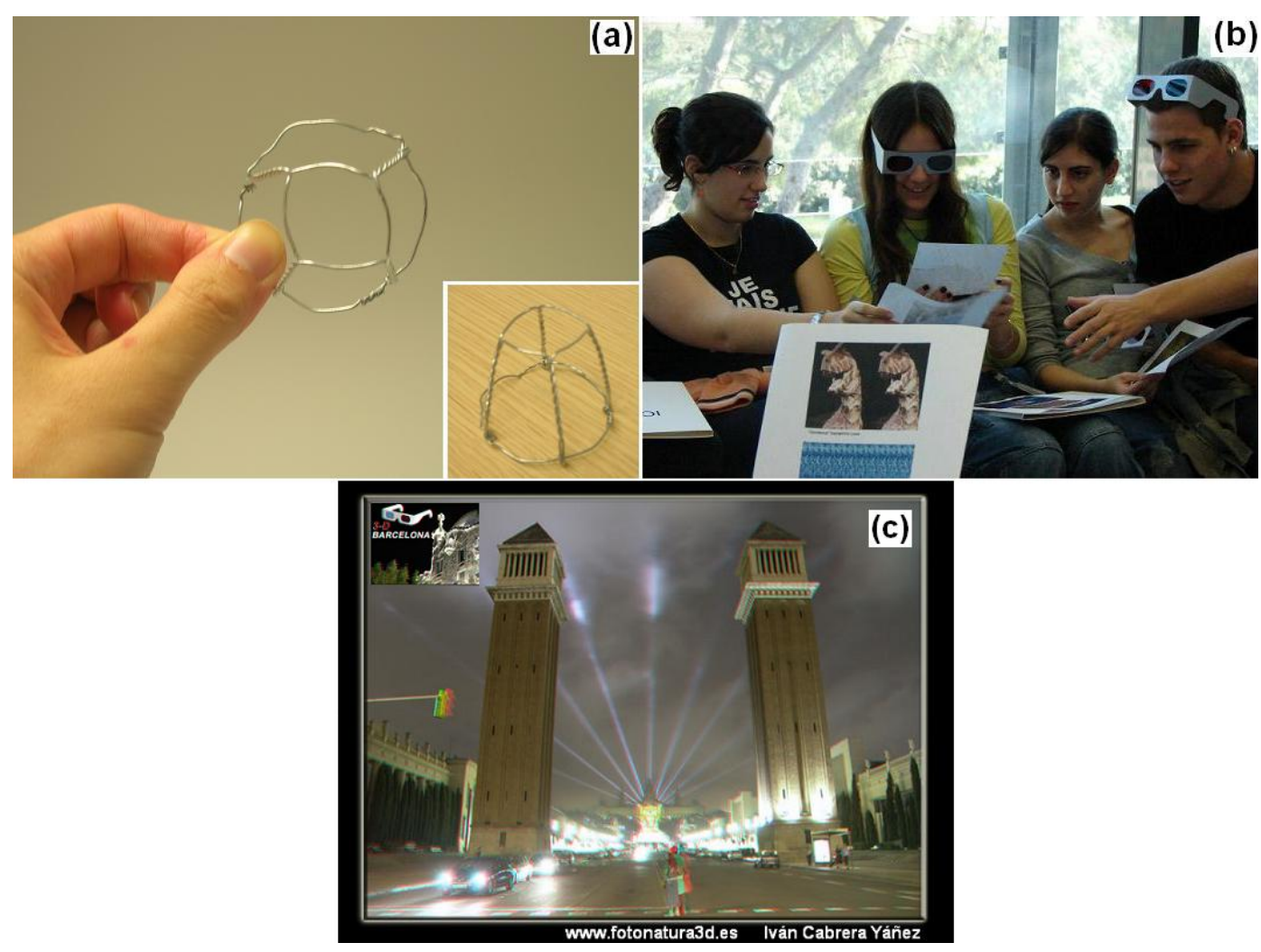

Figure 5: (a) Two rings of metal wire. (b) Demonstration of stereoscopic vision with 3D glasses. (c) An example of $3 \mathrm{D}$ pictures [6].

Table 3: Materials for the demonstration of stereoscopic vision.

\begin{tabular}{|l|c|}
\hline Materials & number \\
\hline Color photos for 3D image & 50 \\
3D glasses & 15 \\
Metal wires with two rings & 5 \\
A projector and a laptop to show 3D movie & 1 \\
\hline
\end{tabular}


3D glasses are such that the right eye see through a red filter and the left one a blue filter. Since these two colors are complementary, it is then possible to include the two pictures on one single 3D picture and print it on a piece of paper. The red picture will only be seen by the left eye with the blue filter. The reason for this is that the right eye with the red filter does not distinguish between red and white. Hence the eyes see two pictures with slightly different angles of the same scene, which enables our brain to give us a sense of depth and a sense of stereoscopic vision. Because of the complementarity of these colors, the same principle also applies to movies. Apart from the internet, we got the 3D pictures through a collaboration with a local photographer who specializes in 3D imaging [6]. The required kit for this session is shown in Table 3.

\subsection{Concluding remarks}

After the demonstrations, students, teachers and volunteers gather and review what the students learn, by asking the three question that we asked at the beginning. Then, we take 5 minutes to get feedback from students who write their opinion on sheets of papers so that we can improve our presentation. Some of the high school teachers who came along with students asked about the materials used in demonstration so that they could perform some of the experiments at school. We also provided them with take-home materials, such as liquid crystal cells and 3D pictures.

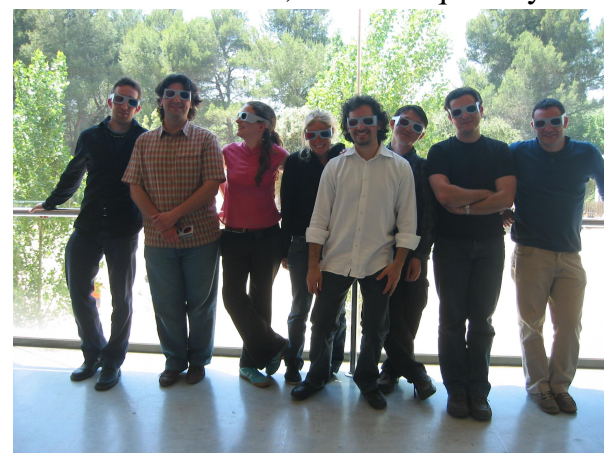

Figure 6: Participants of the first 'The Day of the light' from ICFO OSA student chapter

\section{Conclusion}

This program has the advantage of not being lengthy, which allows school teachers and students to attend it without sacrificing regular curricular activities. The workshop does not require sophisticated equipment so that teachers and students can easily learn how to reproduce the demonstration themselves. With this aim, they are provided with takehome materials, such as liquid crystal cells and 3D pictures, a well appreciated conclusion. Finally, our volunteers are post-graduate students who are close to the age of students so that they feel more friendly and comfortable in comparison to their teachers.

\section{Acknowledgement}

This program is supported by funding by ICFO, OSA, and by the Government of Catalonia (Generalitat de Catalunya). We would like to acknowledge active members who help organize this activity, Xavier Vidal, Clara Ines, Noelia Gonzalez, Maurizio Rhigini, Sandro Perrone, Giorgio Volpe, Sibylle Braungardt, as well Prof Luis Torner (ICFO Director and chapter advisor) Prof. Dmitri Petrov for laboratory tour, Dr Silvia Carrasco, Laura Grau, and Míriam Aguilar for administrative support. We include a snapshot of some of the volunteers for this workshop in Figure 6.

\section{References}

[1] Hannah D. Foreman, Francesca R. Parmigiani, Michal A. F. Roelens, and Robert E. Simpson, "The Lightwave Roadshow" The proceedings of The Education and Training in Optics and Photonics Conference (ETOP), ETOP035, 140 (2005)

[2] Website of ICFO-OSA student chapter, http://osa.icfo.es/activities.php?topic=eldiadelaluz (retrieved April 2, 2007)

[3] Website of ICFO-OSA student chapter, http://osa.icfo.es/ (retrieved April 2, 2007)

[4] Merck KGaA, Germany, http://www.merck.de (retrieved April 2, 2007)

[5] Personal communication with Prof. Stephen D. Jacobs, Laboratory for Laser Energetics, Optics and Chemical Engineering, University of Rochester, Rochester, USA

[6] Iván Cabrerá Yáñez, http://www.fotonatura3D.es (retrieved April 2, 2007) 